\title{
P2Y14 Receptor as a Target for Neutrophilia Attenuation in Severe COVID-19 Cases: From Hematopoietic Stem Cell Recruitment and Chemotaxis to Thrombo-inflammation
}

\author{
Lyvia Lintzmaier Petiz ${ }^{1,2}$ (D) Talita Glaser $^{1}$ (D) $\cdot$ Julio Scharfstein $^{3}$ (D) $\cdot$ Mariusz Z. Ratajczak $^{4}\left(\right.$ D $\cdot$ Henning Ulrich $^{1}$
}

Accepted: 25 January 2021 / Published online: 11 February 2021

(C) The Author(s), under exclusive licence to Springer Science+Business Media, LLC part of Springer Nature 2021

\begin{abstract}
The global SARS-CoV-2 pandemic starting in 2019 has already reached more than 2.3 million deaths. Despite the scientific community's efforts to investigate the COVID-19 disease, a drug for effectively treating or curing patients yet needs to be discovered. Hematopoietic stem cells (HSC) differentiating into immune cells for defense express COVID-19 entry receptors, and COVID-19 infection hinders their differentiation. The importance of purinergic signaling in HSC differentiation and innate immunity has been recognized. The metabotropic P2Y14 receptor subtype, activated by UDP-glucose, controls HSC differentiation and mobilization. Thereon, the exacerbated activation of blood immune cells amplifies the inflammatory state observed in COVID-19 patients, specially through the continuous release of reactive oxygen species and extracellular neutrophil traps (NETs). Further, the P2Y14 subtype, robustly inhibits the infiltration of neutrophils into various epithelial tissues, including lungs and kidneys. Here we discuss findings suggesting that antagonism of the P2Y14 receptor could prevent the progression of COVID-19-induced systemic inflammation, which often leads to severe illness and death cases. Considering the modulation of neutrophil recruitment of extreme relevance for respiratory distress and lung failure prevention, we propose that P2Y14 receptor inhibition by its selective antagonist PPTN could limit neutrophil recruitment and NETosis, hence limiting excessive formation of oxygen reactive species and proteolytic activation of the kallikrein-kinin system and subsequent bradykinin storm in the alveolar septa of COVID-19 patients.
\end{abstract}

Keywords Hematopoietic stem cells · Purinergic signaling · P2Y14 receptor · Neutrophils · COVID-19 · SARS-CoV-2 · Bradykinin storm

\section{Introduction}

The recently discovered SARS-CoV-2 virus has caused a global pandemic with rapid spread, reaching a mortality rate between $2-3 \%$. Since its first reported appearance, COVID19 - the infectious disease caused by SARS-CoV-2 - resulted in over 2.3 million deaths (WHO Coronavirus disease situation reports accessed on February 10, 2021). Disease

This article belongs to the Topical Collection: Special Issue on COVID19 Pandemic and Stem Cells

Guest Editor: Mariusz Z. Ratajczak

Henning Ulrich

henning@iq.usp.br

Lyvia Lintzmaier Petiz

lyviapetiz@usp.br

Talita Glaser

talita.glaser@usp.br

Julio Scharfstein

scharf@biof.ufrj.br

Mariusz Z. Ratajczak

mariusz.ratajczak@louisville.edu
Departamento de Bioquímica, Instituto de Química, Universidade de São Paulo, Av. Prof. Lineu Prestes, 748. Sala 964 Bloco 9 Superior, Cidade Universitária, São Paulo, SP 05508-000, Brazil

2 Instituto Nacional de Ciência e Tecnologia em Medicina Regenerativa, INCT-REGENERA, Rio de Janeiro, Brazil

Instituto de Biofísica Carlos Chagas Filho, Universidade Federal do Rio de Janeiro, Rio de Janeiro, Brazil

4 Stem Cell Institute at James Graham Brown Cancer Center, University of Louisville, Louisville, KY, USA 
symptoms present a wide spectrum, from asymptomatic patients to multi-organ failure and death. While most cases are moderate, severe cases display fast progression to pneumonia, acute respiratory distress syndrome (ARDS) and coagulopathy, among other clinical manifestations [1]. Inflammatory responses during SARS-CoV-2 infection have determinant roles on disease severity. The virus infects type II pneumocytes of the alveolar epithelium by binding the Spike protein, expressed on the virus surface, to the host cell receptor angiotensin-converting enzyme 2 (ACE2).

Hematopoietic stem cells (HSC) differentiate into immune cells, which provide defense against viral infections [2]. Importantly, recent studies suggest that elderly patients may become more susceptible to SARS-CoV-2 infection due to mutations accumulated in HSC during life, that compromise normal HSC proliferation, mobilization and differentiation [3, 4]. Genetically modified HSC giving rise to blood cells with altered characteristics denotes the concept of clonal hematopoiesis. Thus, this phenomenon may lead to proinflammatory profiles that correlate to an increased risk of severe diseases [5]. HSC may also provide a pool for SARS-CoV-2 infection. Ropa and collaborators [2] showed that HSC express the ACE2 and present impaired self-renewal in the presence of SARS-CoV-2. Infected HSC may be eliminated by immune cells, diminishing the stem cell pool and/or raising the immune response against viral infection. In line with SARS-CoV-2 infection influence on HSC biology, other stem cells, such as neural and lung epithelial stem cells, express ACE2 and may also be infected. In this regard, interference of COVID-19 has been proposed to contribute to neurological and respiratory symptoms' manifestation $[6,7]$.

SARS-CoV-2 spike protein/ACE2 interaction may induce hyperactivation of the Nlrp3 inflammasome, being the culprit of the "cytokine storm", and pyroptotic damage of HSC $[8,9]$. Hyperactivation of the Nlrp3 inflammasome leads to cellular release of several mediators, such as cytokines IL-1 $\beta$ and IL18 , and danger-associated molecular pattern molecules (DAMPs) from leaky cells, including mediators of purinergic signaling, as for example extracellular ATP and UDP-glucose [10]. This step initiates expression upregulation of other proinflammatory cytokines, such as TNF- $\alpha$, INF- $\gamma$, IL-6 and IL- 8 , as well as activation of complement and coagulation cascades [9]. The consequence is an immune response consisting of neutrophil infiltration into the lung tissue. The extent of this infiltration contributes to the cytokine storm event, which is frequently considered the initiating cause for severe and fatal COVID-19 cases [11]. Whole blood transcriptome profiling of COVID-19 patients revealed peak levels for most cytokines, except for IL-1, after respiratory function nadir. In view of that, the cytokine storm might not be the primary cause of impaired respiratory function, but its consequence [12]. Such hypothesis is supported by data showing Nlrp3 inflammasome promotion of lung inflammation, mainly through IL- $1 \beta$ production by recruited dormant neutrophils [13].
Purinergic signaling controls many cellular processes, including proliferation, differentiation and cell death. Purinergic receptors are divided into adenosine-activated P1 and ATPand other extracellular nucleotide-activated $\mathrm{P} 2$ receptors. $\mathrm{P} 2$ receptors are further classified into P2X 1-P2X 7 ionotropic and P2Y1,2,4,6,11,12 and 14 G-protein-coupled metabotropic receptors. Regulation of $\mathrm{P} 1$ and $\mathrm{P} 2$ receptor activity occurs through ectonucleotidases, which degrade ATP into adenosine. Adenosine is further inactivated into inosine by adenosine deaminase (see [14] for a complete review on the purinergic signaling system). Purinergic receptors participate in development, stem cell differentiation and tissue repair [15]. Lately, special attention has been given to purinergic receptors in regulating hematopoiesis, such as promotion of diurnal HSC liberation into the peripheral blood [16]. Purinergic signaling is essential for priming danger signals, which are translated through HSC signals into proinflammatory signals. For this, purinergic mobilization of the HSC niches occurs in response to the pro-mobilization granulocyte colony-stimulating factor (G-CSF) [17]. The P2X 7 receptor expressed on hematopoietic progenitor cells becomes activated, secreting more ATP and immune mediators including TNF- $\alpha$, IFN- $\alpha$ and IFN- $\gamma$ [18]. This receptor is largely involved in innate immunity and inflammasome activation, as previously reviewed $[16,18]$. Further purinergic receptors expressed by HSC include the P2Y14 receptor (P2Y14R) $[19,20]$, whose activation might enhance immune cell mobilization following SARS-CoV-2 infection. The P2Y14R defines distinct lineages of HSC (P2Y14 ${ }^{+} \mathrm{Lin}^{-}$vs. P2Y14 ${ }^{+}$Sca$1^{+}$) with different population capabilities [20]. These features may be important for the understanding of how P2Y14R action on HSC differentiation and mobilization contributes to neutrophil recruitment and activation defining severe COVID-19 pathology.

\section{Neutrophils in COVID-19}

Neutrophils, the most abundant type of granulocytes, are essential short-living cells (6-12 hours) of the innate immune system. Thus, they are produced from HSC at a rate of approximately 10 billion cells a day [21]. Neutrophil production and elimination must be strictly regulated, since low levels of neutrophils (neutropenia) result in recurrent infection and systemic inflammation, while high levels of neutrophils (neutrophilia) also lead to an inflammatory state, due to cytotoxic damage of the healthy tissue [22]. COVID-19 cohort studies reported high levels of neutrophils and sustained low levels of lymphocyte counts in patients, leading to a high neutrophil-to-lymphocyte ratio (NLR), which is predictive for severe illness in the early stage of SARS-CoV2 infection [23]. Lung autopsies of deceased patients displayed neutrophil infiltration into pulmonary capillaries, 
with extravasation into the alveolar area and neutrophilic mucositis. Interestingly, neutrophils are less reactive and adherent in children, which in fact develop less severe forms of COVID-19 [24]. It has been hypothesized that reactive oxygen species (ROS) produced by neutrophil activation would result in a cascade of biological events culminating in COVID-19 severity [25]. The overwhelming production of ROS can result in the release of neutrophil extracellular traps (NETs), web-like structures composed of chromatin containing neutrophil granule proteins, that traps, neutralizes and kills bacteria, fungi and viruses [26]. The formation of NETs primarily occurs through a neutrophil death process named NETosis, and if overproduced, becomes highly cytotoxic and can directly kill epithelial and endothelial cells, contributing to several pathophysiological conditions, like thrombosis, asthma, cystic fibrosis and sepsis [27]. NET-induced necroinflammation plays a central role in cytokine storm development, release of complement proteins, thromboinflammation and multi-organ failure in COVID-19 [28]. Therefore, neutrophilia attenuation is a powerful strategy for prevention of dangerous COVID-19 progression.

\section{Purinergic Signaling in COVID-19}

Purinergic receptors have raised interest as novel targets for COVID-19 treatment [29], as they participate in the regulation of the innate immune system and platelet function, which are highly relevant for hemostasis, thrombosis and inflammatory processes, playing central roles in COVID-19 disease [30]. The literature remains scarce regarding COVID-19 and purinergic signaling, although a few hypotheses were raised recently. P2X7 receptor inhibition has been proposed for cytokine release attenuation in severe pneumonia cases in COVID-19 patients, due to its central role in inflammation and Nlrp3 inflammasome stimulation [31], including the neuroinflammation causing neurological disorders [32]. As mentioned above, the Nlrp3 inflammasome may be directly activated in target cells, like HSC and epithelial progenitor cells, following SARS-CoV-2 Spike protein binding to ACE2 [8]. Activation of the Nlrp3 inflammasome also promotes the release of purinergic signaling mediators, such as extracellular ATP (eATP), activating the P2X7 and other purinergic receptors. Moreover, the $\mathrm{A}_{2 \mathrm{~A}}$ receptor that binds extracellular adenosine (eAdo) may also be important for cell defense mechanisms against COVID-19 [33]. Inhibition of interferon type I (IFN-1) production is a classical strategy that several viruses use to neutralize innate immunity response. eATP plays a crucial role in IFN-1 signaling, although continuous eATP hydrolysis by ectonucleotidases, such as CD73 and CD39, that process eATP to its metabolite eAdo, may turn the host cell more vulnerable. To explain this, eATP commonly promotes immune response activation and inflammation, while
eAdo suppresses immune responses and induces antiinflammatory effects. Suppression of $\mathrm{A}_{2 \mathrm{~A}}$ receptors could preserve extracellular eATP levels, which in turn would favor IFN-1 signaling [33]. Moreover, the ectonucleotidase CD73, that together with CD39 is known as 'immune checkpoint mediators', had a remarkably lower expression in lymphocyte populations of COVID-19 patients [34]. More recently, the $\mathrm{A}_{2 \mathrm{~A}}$ receptor agonist dipyridamole was suggested as a therapeutic option for thrombo-inflammation prevention in COVID-19 patients [35].

\section{The P2Y14 Receptor}

The Gi protein-coupled P2Y14R is known to activate the innate immune system. This receptor is strongly activated by extracellular UDP-sugars, with a relative order of potency of UDPglucose $>$ UDP-galactose $>$ UDP-glucuronic acid $>$ UDP-Nacetylglucosamine, being the only purinergic receptor activated by this type of nucleotides [36]. P2Y14R activation is not only limited to UDP-sugars, as UDP also strongly activates both rodents and human receptors [37]. UDP-glucose is synthesized in the cytosol, although it can be found in large amounts in the endoplasmic reticulum lumen, where it is used as a sugar donor for glycosylation reactions. UDP-sugars do not diffuse back to the cytosol, so it is believed that they are basally released from a wide range of cells during glycoconjugate exocytosis [10]. The pathways resulting from P2Y14R activation are not completely elucidated; however, experimental data showed that extracellular signaling of UDP-glucose induces phosphorylation of ERK1/2 MAP kinases [38].

The human P2Y14R is abundantly expressed in several tissues, including lung, kidney, brain, heart, adipose tissue, skeletal muscle, placenta, spleen, stomach and intestine [36], where it is described to be mainly involved in immune responses and inflammation [39]. Unfortunately, there is no data yet available for P2Y14R differential expression in COVID19 pathology. P2Y14R inhibition decreases neutrophil recruitment and activation in human lung and kidney epithelial cells. When activated, P2Y14R induces mobilization and differentiation of HSC, which are neutrophil precursors [40, 41], as well as regeneration responses [42]. It is highly expressed in neutrophils [43], promoting neutrophil activation [44], and chemotaxis into lung tissue [45]. Hence, it is suggested that P2Y14R activity inhibition may decrease COVID-19-induced neutrophilia (and consequently NLR), managing uncontrolled innate immune system activation observed in severe COVID19 patients.

\section{The P2Y14 Receptor and Hematopoietic Stem Cells}

It was already postulated that stem cells might be an infection site for SARS-CoV-2, making them a possible therapy target 
for COVID-19 treatment [46]. Human derived HSC express ACE2 protein, and when in contact with Spike protein, expand less effectively and form less functional colonies [2]. Like all blood cells, neutrophils are derived from HSC, which are responsible for the hematopoiesis process continuously occurring during life. To maintain a steady turnover, hematopoiesis requires a special environment that provides protection, molecular cues and specific physical interactions for these cells. This compartment is known as the HSC niche [47]. Experimental evidence indicates that neutrophils regulate the hematopoietic niche by providing additional protection to hematopoiesis processes, such as quiescence, regeneration and proliferation [48].

Intriguingly, the P2Y14R affects such processes (Fig. 1). First, the P2Y14R is expressed in a subpopulation of primitive human bone marrow HSC and hematopoietic progenitor cells, detected both in adult and fetal samples. The incubation of this specific subset of cells with conditioned media from bone marrow stroma led to P2Y14R activation followed by chemotaxis [19]. Such effects were blocked by pertussis toxin, a drug that catalyzes ADP-ribosylation of heterotrimeric Gi/oproteins (the G protein to which P2Y14R couples), inactivating the P2Y14R. In stress situations, the adaptability of the hematopoietic system to meet the demand of innate immune cells is remarkable and critical for survival. During stress-induced responses, such as acute infection and inflammatory stress situations, HSC located in the niche are mobilized into the peripheral blood [49]. The P2Y14R agonist UDP-glucose, together with other nucleotides, is released into extracellular fluids in response to stress [10]. In healthy mice, subcutaneous injection of UDP-glucose promoted the mobilization of HSC to the peripheral blood. After being harvested, these HSC proved to be colony-forming cells, indicative of differentiation capabilities [40]. In lethally irradiated mice, UDP-glucose triggered the mobilization of functionally distinct HSC subpopulations. These specific subpopulations displayed superior long-term repopulating capacity compared to cells mobilized by G-CSF, the most clinically used factor for HSC mobilization [40]. In bone marrow-derived cell culture, P2Y14R expression was selectively induced by RANKL (a protein belonging to the tumor necrosis factor family) stimulation during osteoclastogenesis. Further, addition of UDPglucose and other UDP-sugars induced the differentiation of HSC into osteoclasts, which also depended on RANKL activation [41].

The P2Y14R has also important functions in the placenta, a rich source of HSC. The human placenta has one of the highest levels of P2Y14R expression [36]. In mice, Sca-1expressing cells are the population holding HSC activity in the placenta, and $75.2 \%$ of Sca- $1^{+}$cells express the P2Y14R. After purification by fluorescence-activated cell sorting (FACS), P2Y14R ${ }^{+}$Sca- $1^{+}$cells displayed a significantly higher capacity of forming colonies when compared to
$\mathrm{P}^{2} \mathrm{Y}_{14 \mathrm{R}^{-}}$Sca- ${ }^{+}$cells [20]. The regenerative properties of HSC can also be mediated by P2Y14R. Loss of P2Y14R leads to reduced HSC capability of responding to multiple stress factors [42]. In a model of radiation-induced stress, HSC of P2Y14R knockout mice embryos were more prone to enter senescence than wild-type ones were. In adulthood, also after radiation-induced stress, P2Y14R knockout mice lost more bone-marrow cells and developed hypersensitivity to hematological stress compared to wild-type animals, reinforcing the receptor liability on HSC function [42]. The described effects on HSC processes indicate that P2Y14R-dependent modulation is not limited to mature blood cells, like neutrophils, as these effects are already present in progenitor cells.

Chemokine signaling cues play important roles in HSC mobilization. Relevant to this topic, the P2Y14R has considerable amino acid sequence similarities with chemokine receptors $(20-30 \%)$. The similarity is based on the conserved DRYLAC chemokine receptor signature motif [50]. HSC mobilization induced by cytokine IL- 8 release depends on neutrophil activation, reinforcing neutrophils as key regulator of this process [51]. Further, neutrophils can modulate the hematopoietic niche, by promoting regulatory effects on quiescence, proliferation and regeneration (Fig. 1) [48]. The P2Y14R also affects hematopoietic niche processes, and its activation can promote IL-8 release by epithelial cells [52]. Therefore, P2Y14R-induced effects on neutrophils may be responsible for hematopoietic niche regulation. Moreover, lungs and blood from COVID-19 patients showed high levels of IL-8, a cytokine related to both HSC mobilization and the inflammatory cytokine storm [53-55], corroborating the idea that P2Y14R inhibition would prevent excessive neutrophil differentiation and activation in COVID-19 patients.

\section{The P2Y14 Receptor and Neutrophils}

Following mobilization and differentiation, HSC give origin to blood cells, including the ones of the innate immune system, such as neutrophils. UDP-glucose is stable in human neutrophil samples, implying absence or limited activity of ecto-nucleotide pyrophosphatases for UDP-sugars in these cells. Meanwhile neutrophils completely hydrolyze in a CD73- and CD39-dependent manner eATP to eAdo [56]. This indicates that UDP-glucose may accumulate in the extracellular environment, activating the P2Y14R with possible contribution to pathophysiological conditions.

Mature human neutrophils have extremely high levels of functional P2Y14R expression [43], proven by inhibition of forskolin-stimulated cAMP accumulation, as well as the opposing effect observed in the presence of pertussis toxin [44]. Incubation of UDP-glucose with human neutrophils induced cytoskeleton rearrangement, changes of cell shape and increased cell migration, which are features of neutrophil activation. UDP-glucose also promoted a fast, robust and 


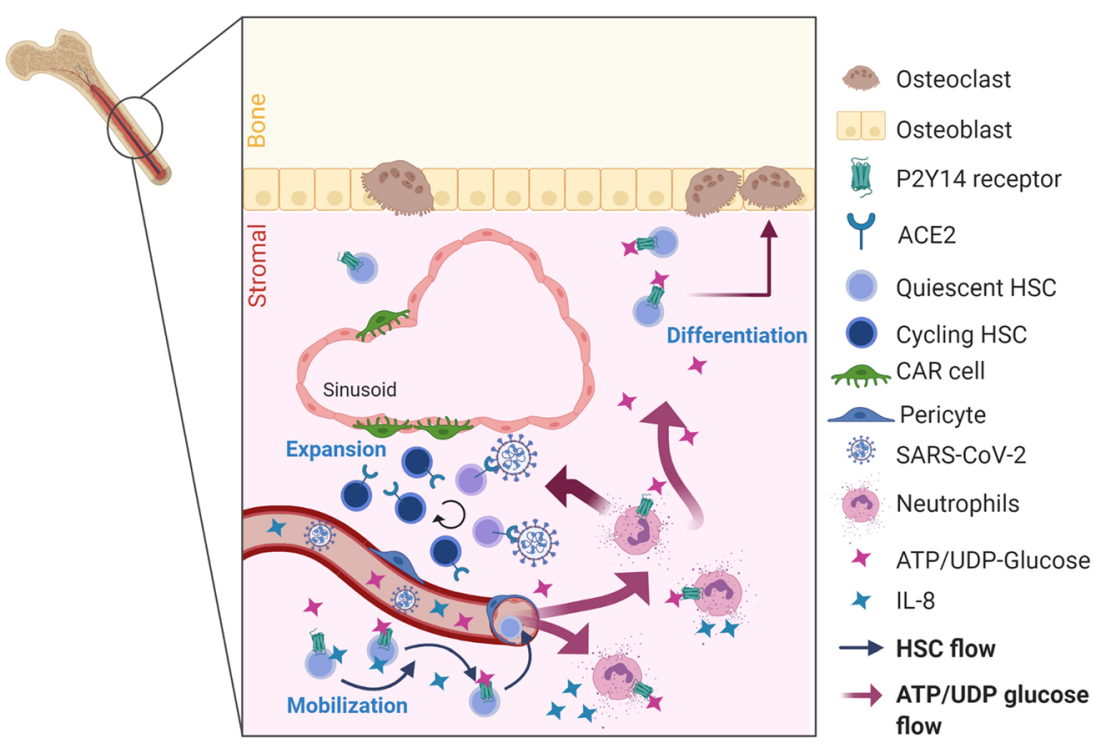

Fig. 1 SARS-CoV-2 infection and P2Y14 receptor interference in hematopoietic stem cell (HSC) homeostasis in the bone marrow. During viral infection, DAMPs, such as ATP/UDP-glucose and Interleukin-8 (IL-8), are released into the blood circulation following tissue injury. The bone marrow presents the niche for HSC and responds to these signaling molecules. In situ activated neutrophils favor expansion and proliferation of HSC that is supported by Cxcl12-abundant reticular
(CAR) cells and pericytes around the sinusoid region, while Spike protein of SARS-CoV-2 directly arrests their cell cycle. In addition, UDP-glucose triggers release of IL- 8 by neutrophils in the bone marrow, inducing mobilization of HSC into the blood flow and consequently increasing the burden of proinflammatory processes in the periphery. Furthermore, activation of the P2Y14 receptor expressed by HSC guides HSC differentiation towards the osteoclast fate. Created with BioRender.com concentration-dependent activation of RhoA, which is the main Rho family isoform found in neutrophils [56]. Rho GTPases are key regulators of cytoskeleton rearrangement necessary for neutrophil functions, such as adhesion, cell polarization, directional migration, phagocytosis, and microbial killing [57]. UDP-glucose promoted both chemotaxis and Rho GTPase activation in differentiated human promyelocytic leukemia cells (HL60), which did not occur in undifferentiated HL60 cells [56]. Accordingly, HL60 cells do not express the P2Y14R, while DMSO-induced differentiation into a neutrophil-like phenotype remarkably promoted expression of this receptor [38], indicating that UDP-glucose activation of Rho was P2Y14R-dependent. Confirming this hypothesis, the expression of recombinant human P2Y14R on undifferentiated HL60 cells promoted UDP-glucose-dependent RhoA activation and chemotaxis [56]. These results indicate a proinflammatory role of P2Y14R activation, leading to human neutrophil chemotaxis. PPTN, the selective high-affinity P2Y14R antagonist, confirms the specifity of P2Y14R for human neutrophil chemotaxis [58]. PPTN treatment blocked UDP-glucose-promoted chemotaxis in neutrophillike differentiated HL60 cells and freshly isolated human neutrophils, corroborating previous results [58]. Relevant to this topic, UDP-glucose promotes P2Y14R-dependent production of IL-8/CXCL8 in human epidermal keratinocytes stimulated with monosodium urate [59]. Since neutrophils migrate to the epithelium as a response to tissue damage, it is likely that the P2Y14R promotes neutrophil chemotaxis during this process.

\section{P2Y14R and Neutrophil Chemotaxis in Lung Epithelial Cells}

While neutrophils are important mediators in the defense against infections, they are also major contributors to the inflammatory response causing lung tissue destruction. Failure to effectively manage inflammation in cystic fibrosis $(\mathrm{CF})$ and chronic obstructive pulmonary disease (COPD) is due, in part, to a limited understanding of the processes responsible for neutrophil recruitment, activation and clearance in diseased airways [60]. In agreement with the tendency of nucleotides to accumulate in extracellular spaces under stress conditions, samples of diluted bronchoalveolar lavages from COPD patients contained 200-400 nM of UDP-sugars (UDP-glucose and UDP-N-acetylglucosamine) [39, 61]. In CF patients, neutrophil counts of sputum samples were significantly higher compared to healthy subjects, besides an 11-fold increase in UDP-glucose levels, a sufficient concentration for P2Y14R activation [45]. Thus, the P2Y14R-mediated increase in neutrophil chemotaxis and its expression in epithelial lung cells suggests that this receptor might be involved in neutrophil recruitment in the lungs.

$\mathrm{P} 2 \mathrm{Y} 14 \mathrm{R}$ expression is detected in human lung tissue, the human alveolar epithelial cell line A549, the human bronchial epithelial cell line BEAS-2B, and fresh collected human alveolar epithelial type II pneumocytes [36, 52]. Receptor functionality was confirmed in these cells by the UDP-glucoseinduced increase of $\mathrm{Ca}^{2+}$ currents, which also triggered a 
robust and selective release of the potent neutrophil chemoattractant CXCL8/IL-8 [52]. Both phenomena were inhibited by pertussis toxin. IL-8 release promotes a rapid mobilization of HSC, an event that requires in vivo activation by neutrophils [51], and supposedly correlates with P2Y14R activation [40]. The human lung cancer cell line Calu-3, when stimulated with ionomycin to increase intracellular $\mathrm{Ca}^{2+}$ concentration, secreted mucin containing UDP-glucose [62]. SPOC1 rat tracheal epithelial cells, which are mucinproducing mucous goblet cells, also released UDP-glucose when stimulated with extracellular ATP, which commonly promotes cellular activation and inflammation [62]. Studies with PPTN confirmed that UDP-glucose-induced effects on lung inflammation depend on P2Y14R activation. This was observed in healthy mice [45] and mouse inflammation models, such as the CF-like $\beta \mathrm{ENaC}$ transgenic mice [45], allergen challenge [63] and lipopolysaccharide (LPS) models [64]. In vivo instillation of UDP-glucose into wild-type mouse trachea resulted in enhanced neutrophil lung recruitment, which was nearly abolished in the presence of PPTN. $\mathrm{ENaC}$-overexpressing $\beta \mathrm{ENaC}$ transgenic mice, used as $\mathrm{CF}$ model due to their characteristic mucin hypersecretion, revealed reduced neutrophil lung inflammation after PPTN administration [45]. P2Y14R relevance in immune system activation in allergen-induced stress was confirmed by a significant upregulation of its expression in airway epithelial cells, neutrophils and eosinophils after house dust mite (HDM) challenge [63]. Lastly, PPTN blocked neutrophil lung recruitment after intranasal LPS administration, indicating that LPSinduced chemotaxis depended on P2Y14R activation [64]. These results indicate P2Y14R-mediated effects of UDPglucose as a local mediator of neutrophil chemotaxis and activation. Thus, considering the P2Y14R role in neutrophils and chemotaxis in lung epithelia, its inhibition through PPTN could decrease neutrophil hyperactivation, preventing the cytokine storm observed in critically ill COVID-19 patients (Fig. 2).

\section{P2Y14 Receptor and Neutrophil Chemotaxis in Kidney Epithelial Cells}

In the virus-infected epithelia, the activation of neutrophils and the continuous release of cytokines, like IL-1 and TNF, starts an amplification loop that supports cytokine storm. The consequent release of further proinflammatory factors leads to thrombotic/fibrinolytic alterations, causing thrombosis in arteries and microvasculature of organs like heart and kidneys [65]. Patients with respiratory failure/ARDS are at increased risk of associated kidney injury (AKI), which makes AKI strongly correlated to severe cases of COVID-19 [66]. Post-mortem biopsies from COVID-19 patients showed varying degrees of acute tubular necrosis. The same kidney samples tested negative for SARS-CoV-2 presence, emphasizing that over activated systemic inflammation causes kidney failure in deadly cases [67]. Besides inflammatory and immune-mediated effects, COVID-19-induced pathophysiological processes, such as impaired gas exchange and hemodynamic damage, increase the risk of AKI [68], reinforcing the cross-talk between lung and kidneys in critically ill COVID-19 patients.

A major cause of AKI seems to be neutrophil infiltration that triggers the cytokine storm and later induces kidney injury. Kidney collecting duct intercalated cells present high levels of P2Y14R, that upon binding of UDP-glucose, promotes production of chemokines. Release of these factors leads to neutrophil infiltration and renal inflammation, therefore contributing to the onset of AKI [69]. This finding corroborates other studies, which observed the same effect of UDP-glucose on intercalated cells, such as P2Y14R activation and recruitment of neutrophils through cytokine release. UDP-glucose-promoted proinflammatory responses were found in a renal epithelial cell model, denominated Madin-Darby Canine Kidney subclone C11 cell line (MDCK-C11). Activation of the MAPK-MEK1/2-ERK1/2 pathway in MDCK-C11 cells mediated production of inflammatory cytokines, which was prevented by inhibition of the P2Y14R by PPTN [70]. Battistone and collaborators [71] proved that early AKI induced by ischemia in mice could be prevented by P2Y14R antagonism by PPTN or through P2Y14R gene ablation in renal intercalated cells. Both strategies were capable of inhibiting chemokine expression, lessening neutrophil and monocyte renal infiltration, thus preventing proximal tubule damage and kidney dysfunction. Moreover, they detected high levels of UDP-glucose in patients at intensive care units, corroborating that UDP-glucose is released under stress conditions [10]. We believe that the UDP-glucose/ P2Y14R axis is a potential target for attenuation of extreme kidney failure and death of COVID-19 patients induced by systemic inflammation. Selective P2Y14R antagonism is supposed to avoid neutrophil infiltration in both lungs and kidneys.

\section{Targeting the P2Y14R: Limiting Crosstalk Between Complement and Kallikrein-kinin Systems}

COVID-19 was initially characterized as a severe respiratory disease caused by the misfiring of the immune response [72]. However, recent findings point to the correlation between systemic thrombo-inflammatory responses and massive tissue infiltration by neutrophils and monocytes. While a robust innate immunity is capable of controlling SARS-CoV-2 infection in the upper airway tissues of most patients, high risk groups display a weakened antiviral response, unable to prevent the spread of the virus to the lower airways [73].

Following binding to ACE2-expressing type II pneumocytes, SARS-CoV-2 subverts antiviral immunity through downregulation of IFN-1-regulated gene transcription and expression upregulation of genes coding for proinflammatory chemokines/cytokines [74]. In a study exploring the 
SARS-CoV-2- Injured alveolus

P2Y14R antagonist treatment

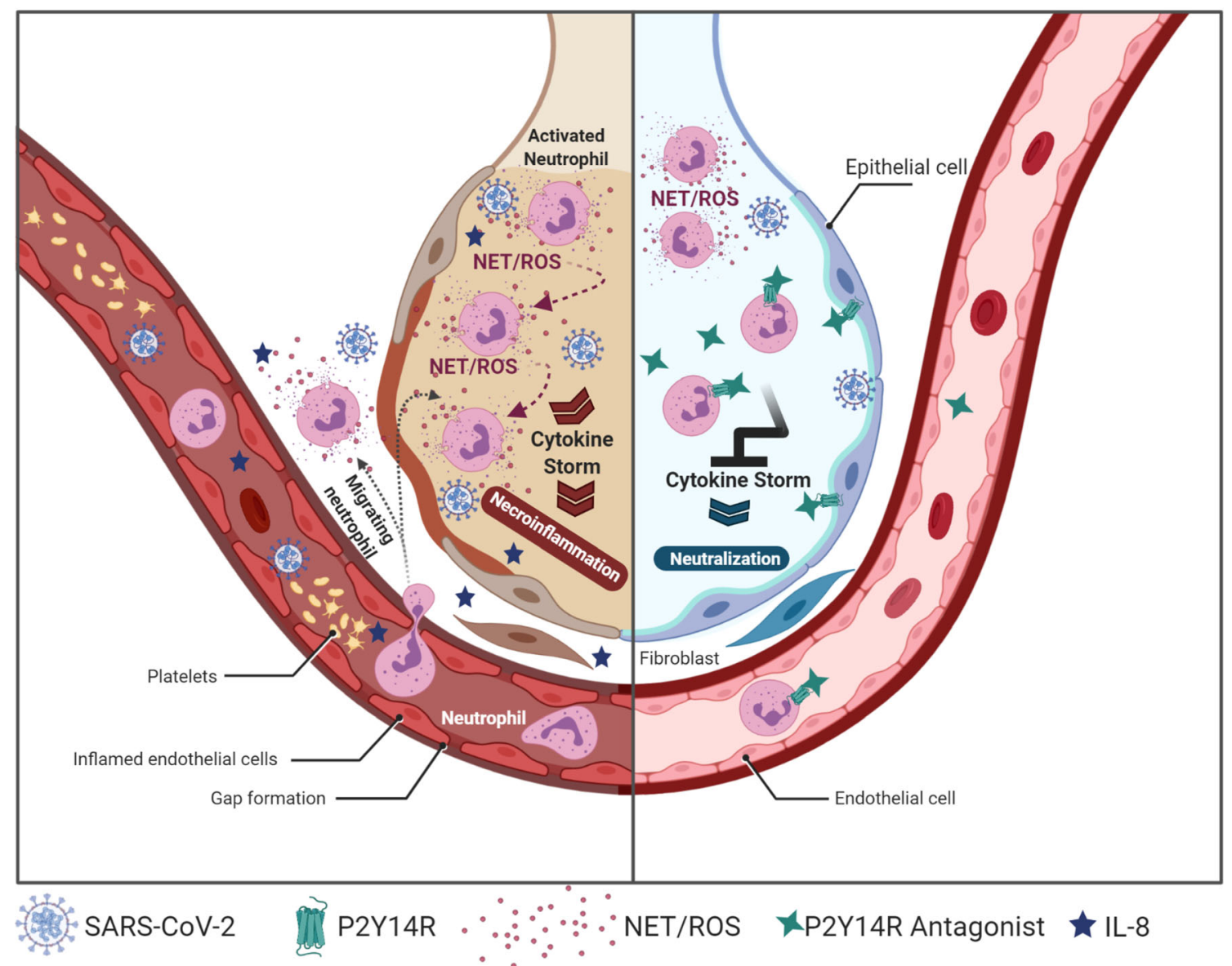

Fig. 2 Inhibition of the P2Y14 receptor could attenuate neutrophilia in COVID-19 patients. Pharmacological modulation of P2Y14R activity may occur by two different ways to prevent neutrophilia, based on its effects on neutrophils and epithelial lung cells. P2Y14R inhibition in the respiratory tissue could mitigate neutrophil recruitment and consequent infiltration into the lungs, at least in part due to release reduction of the

role of the innate response, mainly the complement $\mathrm{C} 5 \mathrm{a} /$ C5aR1 axis in COVID-19 severity, peripheral blood neutrophilia correlated to adverse clinical outcome. Peripheral blood also presented a decrease in the proportion of inflammatory $\mathrm{CD} 14^{\text {low }} \mathrm{CD} 16^{+}$monocytes, raising the possibility that this subset has infiltrated the lung and other tissues [75]. While SARS-CoV-2-induced dynamics of leukocyte recruitment to lower airways is still not clear, it is conceivable that blood plasma leakage may modulate neutrophil and monocyte infiltration through the activation of $\mathrm{C} 3 \mathrm{aR}$ and C5aR1/C5aR2. Dysregulated activation of complement probably occurs due to plasma leakage at the early stage of infection when alveolar septa are overloaded with virus particles. Although the titers of complement-fixing proteins (i.e., Creactive protein, mannose-binding lectin MASP-2 and IgM antibodies against multiple viral antigens) vary between patients, diffusion of complement-fixing proteins through the virus-laden tissues should lead to the extravascular activation potent chemotaxis promotor IL-8 by lung epithelial cells. Further, P2Y14R inhibition could also act directly on neutrophils, avoiding uncontrolled chemotaxis, degranulation and NETosis, thus preventing oxidative stress and the biological cascade that perpetuates neutrophil activation. NETs: neutrophil extracellular traps; ROS: reactive oxygen species. Created with BioRender.com

of the complement system. Complement is also activated as MASP-2 binds to coronavirus N protein [76]. Another mechanism may involve binding of heparan sulfate to the Spike S protein. This interaction constrains the regulatory activity of factor $\mathrm{H}$, an inhibitory complement control protein, on the surface $\mathrm{C} 3$ convertase [77]. Consistent with the idea that complement dysregulation worsens the pathogenic outcome of COVID-19, C5a and proinflammatory cytokines were detected in the bronco-alveolar lavage of COVID-19 patients [75]. Extending the analysis of neutrophil function, COVID-19 patients produced high levels of neutrophils releasing tissue factor and C3 incorporated in NETs [78]. Recently, small independent cohorts of severe COVID-19 patients showed that AMY-101, a compstatin-like cyclic drug efficiently targeting $\mathrm{C} 3 \mathrm{a}$, decreased neutrophil counts and systemically blunted thrombo-inflammation [79].

Pertinent literature for this review, P2Y14R antagonism has a potential role in inhibiting $\mathrm{C} 3 \mathrm{a}$-induced mast cell (MC) 


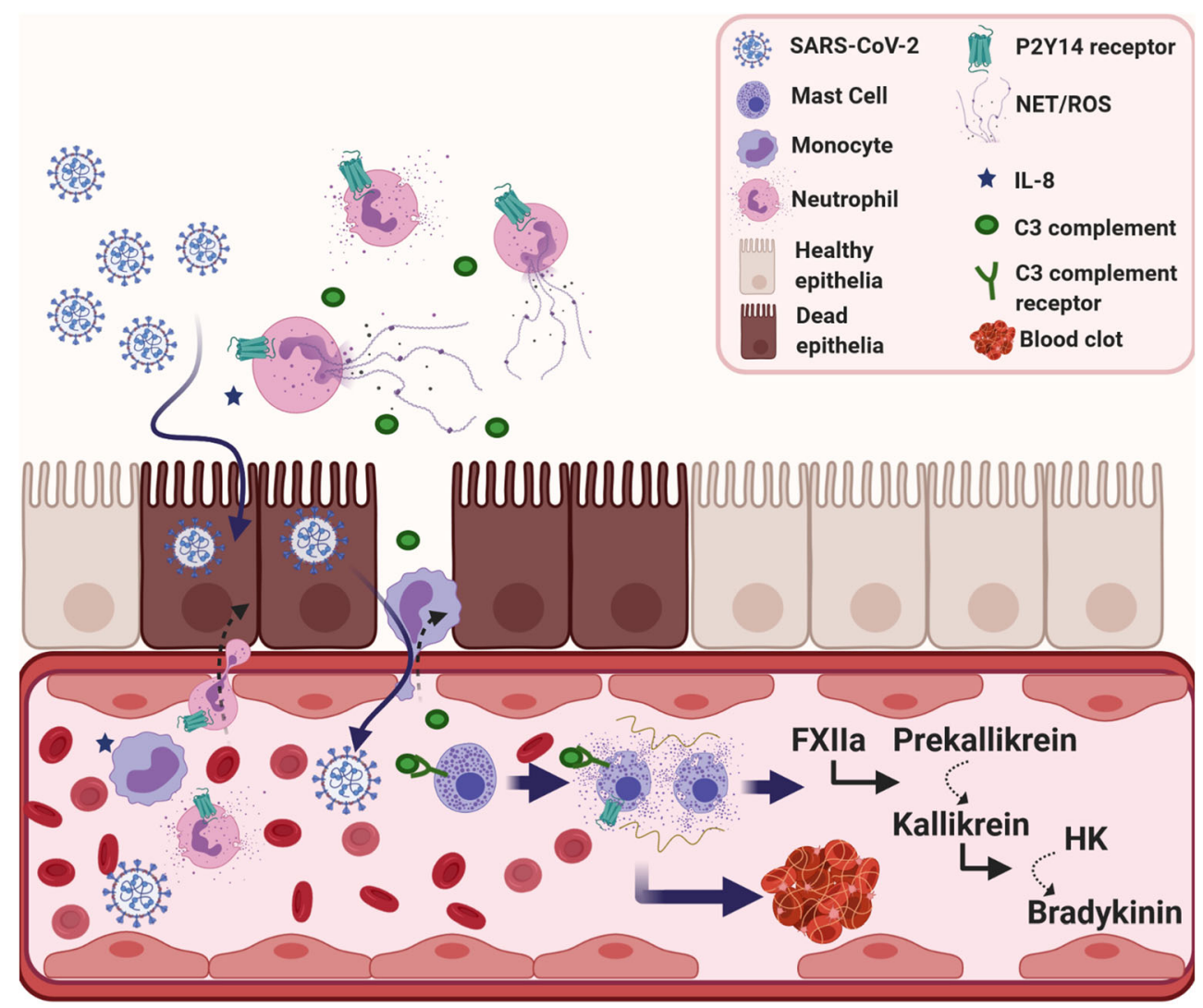

Fig. 3 Participation of mast cell/kallikrein-kinin system overactivation at systemic thrombo-inflammation in COVID-19 patients. The proposed mechanism is based on the interaction between NETs and the complement/coagulation systems. Upon viral infection, cytokines and DAMPs released from dead or dying cells activate endothelial cells, which start to express adhesion molecules, attracting neutrophils and other leukocytes. The release of NETs by neutrophils initiates a broader inflammatory activation, which can disrupt important molecules for endothelial barrier function, promoting plasma leakage. During NETosis, activated neutrophils release NETs to capture and neutralize pathogens, avoiding their spread. NETs involve the $\mathrm{C} 3$ a protein, which in turn activate mast cells. The mast cell degranulation process releases histamine, serine proteases and a great load of heparin. Heparin activates the factor FXII, followed by kallikrein zymogen form to cleavage, which in turn activates the zymogen of bradykinin. The overproduction of bradykinin would then lead to a bradykinin storm. P2Y14 receptors can act upstream in these events, in two different steps: by inhibiting neutrophil activation and subsequently $\mathrm{C} 3 \mathrm{a}$ release; and by blocking mast cell degranulation, decreasing the release of heparin. NETs: neutrophil extracellular traps; ROS: reactive oxygen species; FXII: coagulation factor XII; HK: high molecular weight kininogen. Created with BioRender.com

Bronchoalveolar lavage of COVID-19 patients indicated a decreased expression of ACE, together with increased expression of ACE2, renin, angiotensin, key renin angiotensin system (RAS) receptors, kininogen, several kallikrein enzymes and kinin-B1 and $\mathrm{B} 2$ receptors, when compared to healthy subjects. This differential gene expression indicates elevated bradykinin levels, referred to as "bradykinin storm" [82]. Bradykinin amplifies inflammatory edema via signaling mediated by the kinin-B2 receptor, a $\mathrm{G}$ protein-coupled receptor constitutively expressed in the endothelium. The first example of an intracellular pathogen that increments its infectivity at expense of MC/Kinin-Kallikrein System-driven formation of inflammatory edema (bradykinin-storm) came from studies in animal infected by Trypanosoma cruzi, the parasitic protozoan that causes human Chagas heart disease [83, 84]. Bradykinin-induced inflammatory cascades may worsen ARDS by stimulating excessive formation of hyaluronic acid (hydrogel), that inhibits oxygen exchange in the alveoli of 
COVID-19 [82]. Supporting this hypothesis, treatment of COVID-19 patients with the kinin-B2 receptor selective antagonist Icatibant alleviated ARDS in a study involving a limited cohort [85].

Furthermore, histopathological studies conducted in Brazil revealed that MC density was increased in postmortem biopsies (alveolar septa) of COVID-19 patients, but not in biopsies from patients that died of H1N1 pneumonia [86]. Interestingly, increased MC density in COVID-19 biopsies correlated with higher scores for IL-4, suggesting that the expression profile in alveolar septa of fatal cases might shift to type-2 immune response cytokines, as indicated by transcriptional analyses of peripheral immune responses [72]. Thus, exacerbation of type-2 immune response also leads to degranulation of the MC [87]. Studies in animal models of COVID-19 may clarify the nature of signs promoting MC progenitor recruitment to the injured lung. Pertinently, P2Y14R antagonism has a potential role in inhibiting C3ainduced $\mathrm{MC}$ degranulation, which consequently would prevent NETs formation. Indeed, C3 knock-out mice do not form NETs, reinforcing the influence of the complement system on NETosis [88]. Based on this evidence, an interesting question to investigate is, whether PPTN would be capable of inhibiting $\mathrm{C} 3 \mathrm{a} / \mathrm{C} 5 \mathrm{a}$-driven neutrophilic infiltration and/or thrombo-inflammatory responses elicited by NETs. Figure 3 provides a scheme of neutrophil activation and complement/ coagulation system interactions.

Finally, P2Y14R may have an important role in MC immune response. Briefly, P2Y14R promotes MC degranulation, at least in part through a synergic action with $\mathrm{C} 3 \mathrm{a}$ anaphylatoxin [80], an important component of the complement cascade. Gao and collaborators [89] reported that P2Y14R expression in the MC-like rat basophilic leukemia RBL-2H3 cell line was about 150-fold higher than that of other P2Y receptors (P2Y2, P2Y4 and P2Y6). UDP-glucose addition to RBL-2H3 cells induced MC degranulation, measured by the release of the phospholipid hydrolase $\beta$ hexosaminidase, via activation of pertussis toxin-sensitive calcium transients [89]. In the human MC cell line LAD2, UDPglucose or the synthetic P2Y14R agonist MRS2690 alone did not induce any significant changes in $\beta$-hexosaminidase levels. However, both P2Y14R agonists enhanced C3a-induced $\beta$-hexosaminidase secretion, and stimuli were blunted by PPTN, pertussis toxin and P2Y14R RNA interference [80]. Noteworthy, two asthma genome-wide association studies used to identify risk-associated genes classified $\mathrm{P} 2 \mathrm{Y} 14$ gene as associated with asthma risk, since P2Y14R expression was upregulated more than 3-fold in lung samples of a model of acute allergic asthma [63]. Mice exposed to UDP-glucose induced the release of IL-33, a member of the IL-1 cytokine family usually produced by epithelial cells, endothelial cells and smooth muscle. Once secreted, this alarmin must be converted into a high-affinity ligand for ST2 by neutrophil proteases before activating $\mathrm{MC}$ in an autocrine manner [63]. Based on this collection of findings, it will be worth assessing whether P2Y14R antagonism will suppress MC degranulation, consequently blunting the bradykinin storm at the alveolar septum.

\section{Conclusions}

Here, we propose a mechanism involving the P2Y14R inhibition to attenuate neutrophilia and prevent COVID-19 progression to severe cases. P2Y receptors are important therapeutic targets and often associated with several disorders. P2Y12R antagonists (clopidorgel, prasugrel, ticagrelor and cangrelor) have already gone through clinical trials and are approved for therapy, being applied for at least two decades to prevent thrombosis in patients at risk of heart attack [90]. There are currently no clinical trials for the P2Y14R antagonist PPTN, although preclinical studies in rodent animal models displayed a safe use of PPTN, in both healthy mice and CF-like lung disease mice model [45]. Recently, new P2Y14R antagonists were synthesized and successfully tested in a mouse model of chronic pain [91], indicating that there are alternatives to PPTN for therapeutic applications. Our hypothesis is that the P2Y14R antagonist at low doses would allow neutrophil activation for SARS-CoV-2 removal, while preventing uncontrolled activation and lung infiltration by these cells. Furthermore, it could prevent exacerbated MC degranulation, thus dampening the bradykinin storm and thrombo-inflammation. By preventing cytokine and bradykinin storms, COVID-19 patients would have a lower risk of multi-organ failure due to systemic inflammation. In conclusion, we postulate that novel targets to control neutrophil recruitment are of extreme relevance for respiratory distress and lung failure prevention. Uncontrolled neutrophil chemotaxis observed in COVID-19 could be prevented by P2Y14R inhibition, consequently preventing lung infiltration, cytokine storm, systemic inflammation and severe illness and reducing death rate of intensive care patients. The same applies to damage of other organs such as kidney, where neutrophil infiltrates worsen prognosis clinical outcomes.

Author Contributions LLP and HU developed the central opinion. TG contributed with stem cells and purinergic signaling knowledge; JS contributed with thrombo-inflammation and complement system knowledge; and MZR contributed with hematopoietic stem cells and inflammasome knowledge. All the authors contributed to the writing. HU was the responsible for the invitation and managing of the field experts.

Funding LLP is grateful for a CNPq DTI-A fellowship (CNPq 380405/ 2020-2) awarded by the Instituto Nacional de Ciência e Tecnologia em Medicina Regenerativa, Brazil. TG is grateful for postdoctoral fellowships granted by FAPESP (2015/13345-1-PD). JC acknowledges grant support from FAPERJ/Temático, PRONEX, CNPq Rede COVID-Rio. MZR's work was supported by the NIH grant 2R01 DK074720, the Stella and Henry Hoenig Endowment. HU acknowledges grant support from the São Paulo Research Foundation (FAPESP, 2018/07366-4) and 
the National Council for Scientific and Technological Development for fellowship support (CNPq, project No. 306392/2017-8).

Data Availability Not applicable.

Code Availability Not applicable.

\section{Declarations}

Conflict of Interests/Competing Interests The authors declare no conflict of interest or competing interests.

Ethical Approval Not applicable.

Consent to Participate Not applicable.

Consent for Publication All authors declared their consent for publication.

\section{References}

1. Wu, C., et al. (2020). Risk factors associated with acute respiratory distress syndrome and death in patients with coronavirus disease 2019 pneumonia in Wuhan, China. JAMA Internal Medicine, 180(7), 934-943.

2. Ropa, J., et al. (2020) Human hematopoietic stem, progenitor, and immune cells respond ex vivo to SARS-CoV-2 spike protein. Stem Cell Reviews and Reports, 1-13.

3. Bolton, K. L., et al. (2020) Clonal hematopoiesis is associated with risk of severe Covid-19. medRxiv. https://doi.org/10.1101/2020.11. 25.20233163.

4. Hameister, E., et al. (2020). Clonal hematopoiesis in hospitalized elderly patients with COVID-19. Hemasphere, 4(4), e453.

5. Jaiswal, S., \& Ebert, B. L. (2019) Clonal hematopoiesis in human aging and disease. Science, 366(6465), eaan4673.

6. Zhang, B. Z., et al. (2020). SARS-CoV-2 infects human neural progenitor cells and brain organoids. Cell Research, 30(10), 928931.

7. Valyaeva, A. A., et al. (2020). Expression of SARS-CoV-2 entry factors in lung epithelial stem cells and its potential implications for COVID-19. Scientific Reports, 10(1), 17772.

8. Ratajczak, M. Z., et al. (2020) SARS-CoV-2 entry receptor ACE2 is expressed on very small CD45(-) precursors of hematopoietic and endothelial cells and in response to virus spike protein activates the Nlrp3 inflammasome. Stem Cell Reviews and Reports, 1-12.

9. Ratajczak, M. Z., \& Kucia, M. (2020). SARS-CoV-2 infection and overactivation of Nlrp3 inflammasome as a trigger of cytokine "storm" and risk factor for damage of hematopoietic stem cells. Leukemia, 34(7), 1726-1729.

10. Lazarowski, E. R., et al. (2003). Release of cellular UDP-glucose as a potential extracellular signaling molecule. Molecular Pharmacology, 63(5), 1190-1197.

11. Winkler, E. S., et al. (2020). SARS-CoV-2 infection of human ACE2-transgenic mice causes severe lung inflammation and impaired function. Nature Immunology, 21(11), 1327-1335.

12. Ong, E. Z., et al. (2020). A dynamic immune response shapes COVID-19 progression. Cell Host \& Microbe, 27(6), 879-882.e2.

13. Tian, X., et al. (2017). NLRP3 inflammasome mediates dormant neutrophil recruitment following sterile lung injury and protects against subsequent bacterial pneumonia in mice. Frontiers in Immunology, 8, 1337.
14. Burnstock, G. (2006). Historical review: ATP as a neurotransmitter. Trends in Pharmacological Sciences, 27(3), 166-176.

15. Burnstock, G., \& Ulrich, H. (2011). Purinergic signaling in embryonic and stem cell development. Cellular and Molecular Life Sciences, 68(8), 1369-1394.

16. Adamiak, M., et al. (2020). Novel evidence that purinergic signaling - Nlrp3 inflammasome axis regulates circadian rhythm of hematopoietic stem/progenitor cells circulation in peripheral blood. Stem Cell Reviews and Reports, 16(2), 335-343.

17. Adamiak, M., et al. (2018). Correction: Mobilization studies in mice deficient in sphingosine kinase 2 support a crucial role of the plasma level of sphingosine-1-phosphate in the egress of hematopoietic stem progenitor cells. Oncotarget, 9(75), 34189.

18. Rossi, L., et al. (2012). The sixth sense: hematopoietic stem cells detect danger through purinergic signaling. Blood, 120(12), 23652375.

19. Lee, B. C., et al. (2003). P2Y-like receptor, GPR105 (P2Y14), identifies and mediates chemotaxis of bone-marrow hematopoietic stem cells. Genes Dev, 17(13), 1592-1604.

20. Kook, S. H., et al. (2017). The expression of P2Y14, a purinergic Gprotein coupled receptor, defines functionally distinct subpopulations in placenta-derived hematopoietic stem progenitor cells. Leukemia, 31(12), 2837-2841.

21. von Vietinghoff, S., \& Ley, K. (2009). IL-17A controls IL-17F production and maintains blood neutrophil counts in mice. Journal of Immunology, 183(2), 865-873.

22. Kruger, P., et al. (2015). Neutrophils: Between host defence, immune modulation, and tissue injury. PLoS Pathogens, 11(3), e1004651.

23. Zhu, S., Dong, L., \& Cai, W. (2020). Predictive value of neutrophil to lymphocyte and platelet to lymphocyte ratio in COVID-19. Critical Care, 24(1), 532.

24. Wu, H., et al. (2020). Clinical and immune features of hospitalized pediatric patients with coronavirus disease 2019 (COVID-19) in Wuhan, China. JAMA Network Open, 3(6), e2010895.

25. Laforge, M., et al. (2020). Tissue damage from neutrophil-induced oxidative stress in COVID-19. Nature Reviews. Immunology, 20(9), 515-516.

26. Papayannopoulos, V. (2018). Neutrophil extracellular traps in immunity and disease. Nature Reviews. Immunology, 18(2), 134-147.

27. Villanueva, E., et al. (2011). Netting neutrophils induce endothelial damage, infiltrate tissues, and expose immunostimulatory molecules in systemic lupus erythematosus. Journal of Immunology, 187(1), 538-552.

28. Tomar, B., et al. (2020). Neutrophils and neutrophil extracellular traps drive necroinflammation in COVID-19. Cells, 9(6), 1383.

29. Cardoso, A. M. (2020). COVID-19 and purinergic signaling: the need for investigation. Purinergic Signal, 16(3), 451-452.

30. Cekic, C., \& Linden, J. (2016). Purinergic regulation of the immune system. Nature Reviews. Immunology, 16(3), 177-192.

31. Di Virgilio, F., et al. (2020). A rationale for targeting the P2X7 receptor in Coronavirus disease. British Journal of Pharmacology, 177(21), 4990-4994.

32. Ribeiro, D. E., et al. (2020) Hyperactivation of $\mathrm{P} 2 \times 7$ receptors as a culprit of COVID-19 neuropathology. Molecular Psychiatry. https://doi.org/10.1038/s41380-020-00965-3.

33. Abouelkhair, M. A. (2020). Targeting adenosinergic pathway and adenosine $\mathrm{A}(2 \mathrm{~A})$ receptor signaling for the treatment of COVID19: A hypothesis. Medical Hypotheses, 144, 110012.

34. Ahmadi, P., et al. (2020). Defining the CD39/CD73 axis in SARSCoV-2 infection: the CD73(-) phenotype identifies polyfunctional cytotoxic lymphocytes. Cells, 9(8), 1750.

35. Kanthi, Y., et al. (2020). New (re)purpose for an old drug: purinergic modulation may extinguish the COVID-19 thromboinflammatory firestorm. JCI Insight, 5(14), e140971. 
36. Chambers, J. K., et al. (2000). A G protein-coupled receptor for UDP-glucose. The Journal of Biological Chemistry, 275(15), 10767-10771.

37. Carter, R. L., et al. (2009). Quantification of Gi-mediated inhibition of adenylyl cyclase activity reveals that UDP is a potent agonist of the human P2Y14 receptor. Molecular Pharmacology, 76(6), 1341-1348.

38. Fricks, I. P., et al. (2009). Gi-dependent cell signaling responses of the human P2Y14 receptor in model cell systems. The Journal of Pharmacology and Experimental Therapeutics, 330(1), 162-168.

39. Lazarowski, E. R., \& Harden, T. K. (2015). UDP-sugars as extracellular signaling molecules: cellular and physiologic consequences of P2Y14 receptor activation. Molecular Pharmacology, 88(1), $151-160$.

40. Kook, S., et al. (2013). The nucleotide sugar UDP-glucose mobilizes long-term repopulating primitive hematopoietic cells. The Journal of Clinical Investigation, 123(8), 3420-3435.

41. Lee, S. A., Park, J. H., \& Lee, S. Y. (2013). Selective induction of P2Y14 receptor by RANKL promotes osteoclast formation. Molecular Cell, 36(3), 273-277.

42. Cho, J., et al. (2014). Purinergic $\mathrm{P}_{2} \mathrm{Y}_{14}$ receptor modulates stressinduced hematopoietic stem/progenitor cell senescence. The Journal of Clinical Investigation, 124(7), 3159-3171.

43. Moore, D. J., et al. (2003). GPR105, a novel Gi/o-coupled UDPglucose receptor expressed on brain glia and peripheral immune cells, is regulated by immunologic challenge: possible role in neuroimmune function. Brain Research. Molecular Brain Research, 118(1-2), 10-23.

44. Scrivens, M., \& Dickenson, J. M. (2006). Functional expression of the P2Y14 receptor in human neutrophils. European Journal of Pharmacology, 543(1-3), 166-173.

45. Sesma, J. I., et al. (2016). UDP-glucose promotes neutrophil recruitment in the lung. Purinergic Signal, 12(4), 627-635.

46. Ulrich, H., \& Pillat, M. M. (2020). CD147 as a target for COVID19 treatment: suggested effects of azithromycin and stem cell engagement. Stem Cell Reviews and Reports, 16(3), 434-440.

47. Pinho, S., \& Frenette, P. S. (2019). Haematopoietic stem cell activity and interactions with the niche. Nature Reviews. Molecular Cell Biology, 20(5), 303-320.

48. Cossío, I., Lucas, D., \& Hidalgo, A. (2019). Neutrophils as regulators of the hematopoietic niche. Blood, 133(20), 2140-2148.

49. Batsivari, A., et al. (2020). Dynamic responses of the haematopoietic stem cell niche to diverse stresses. Nature Cell Biology, 22(1), 7-17.

50. Youn, B. S., et al. (1997). Molecular cloning and characterization of a cDNA, CHEMR1, encoding a chemokine receptor with a homology to the human C-C chemokine receptor, CCR-4. Blood, 89(12), 4448-4460.

51. Pruijt, J. F., et al. (2002). Neutrophils are indispensable for hematopoietic stem cell mobilization induced by interleukin- 8 in mice. Proceedings of the National Academy of Sciences of the United States of America, 99(9), 6228-6233.

52. Müller, T., et al. (2005). The P2Y14 receptor of airway epithelial cells: coupling to intracellular Ca2 + and IL-8 secretion. American Journal of Respiratory Cell and Molecular Biology, 33(6), 601609.

53. Bonig, H., \& Papayannopoulou, T. (2012). Mobilization of hematopoietic stem/progenitor cells: general principles and molecular mechanisms. Methods in Molecular Biology, 904, 1-14.

54. de Kruijf, E. F. M., Fibbe, W. E., \& van Pel, M. (2020). Cytokineinduced hematopoietic stem and progenitor cell mobilization: unraveling interactions between stem cells and their niche. Annals of the New York Academy of Sciences, 1466(1), 24-38.

55. Del Valle, D. M., et al. (2020). An inflammatory cytokine signature predicts COVID-19 severity and survival. Nature Medicine, 26(10), 1636-1643.
56. Sesma, J. I., et al. (2012). The UDP-sugar-sensing P2Y(14) receptor promotes Rho-mediated signaling and chemotaxis in human neutrophils. American Journal of Physiology. Cell Physiology, 303(5), C490-C498.

57. Sit, S. T., \& Manser, E. (2011). Rho GTPases and their role in organizing the actin cytoskeleton. Journal of Cell Science, 124(Pt 5), 679-683.

58. Barrett, M. O., et al. (2013). A selective high-affinity antagonist of the P2Y14 receptor inhibits UDP-glucose-stimulated chemotaxis of human neutrophils. Molecular Pharmacology, 84(1), 41-49.

59. Uratsuji, H., et al. (2016). Monosodium urate crystals induce functional expression of P2Y14 receptor in human keratinocytes. The Journal of Investigative Dermatology, 136(6), 1293-1296.

60. Twaddell, S. H., et al. (2019). The emerging role of neutrophil extracellular traps in respiratory disease. Chest, 156(4), 774-782.

61. Sesma, J. I., et al. (2009). Endoplasmic reticulum/golgi nucleotide sugar transporters contribute to the cellular release of UDP-sugar signaling molecules. The Journal of Biological Chemistry, 284(18), 12572-12583.

62. Kreda, S. M., et al. (2007). Coordinated release of nucleotides and mucin from human airway epithelial Calu-3 cells. The Journal of Physiology, 584(Pt 1), 245-259.

63. Ferreira, M. A., et al. (2017). Gene-based analysis of regulatory variants identifies 4 putative novel asthma risk genes related to nucleotide synthesis and signaling. The Journal of Allergy and Clinical Immunology, 139(4), 1148-1157.

64. Amison, R. T., et al. (2017). Lipopolysaccharide (LPS) induced pulmonary neutrophil recruitment and platelet activation is mediated via the $\mathrm{P} 2 \mathrm{Y}(1)$ and $\mathrm{P} 2 \mathrm{Y}(14)$ receptors in mice. Pulmonary Pharmacology \& Therapeutics, 45, 62-68.

65. Klok, F. A., et al. (2020). Confirmation of the high cumulative incidence of thrombotic complications in critically ill ICU patients with COVID-19: An updated analysis. Thrombosis Research, 191, $148-150$.

66. Sharma, P., et al. (2020). COVID-19-associated kidney injury: a case series of kidney biopsy findings. Journal of the American Society of Nephrology, 31(9), 1948-1958.

67. Cheng, Y., et al. (2020). Kidney disease is associated with inhospital death of patients with COVID-19. Kidney International, 97(5), 829-838.

68. Joannidis, M., et al. (2020). Lung-kidney interactions in critically ill patients: consensus report of the acute disease quality initiative (ADQI) 21 workgroup. Intensive Care Medicine, 46(4), 654-672.

69. Breton, S., \& Brown, D. (2018). Novel proinflammatory function of renal intercalated cells. Annals of Nutrition \& Metabolism, 72(Suppl 2), 11-16.

70. Azroyan, A., et al. (2015). Renal intercalated cells sense and mediate inflammation via the P2Y14 receptor. PLoS One, 10(3), $\mathrm{e} 0121419$.

71. Battistone, M. A., et al. (2020). Proinflammatory P2Y14 receptor inhibition protects against ischemic acute kidney injury in mice. The Journal of Clinical Investigation, 130(7), 3734-3749.

72. Lucas, C., et al. (2020). Longitudinal analyses reveal immunological misfiring in severe COVID-19. Nature, 584(7821), 463-469.

73. Florindo, H. F., et al. (2020). Immune-mediated approaches against COVID-19. Nature Nanotechnology, 15(8), 630-645.

74. Blanco-Melo, D., et al. (2020). Imbalanced host response to SARSCoV-2 drives development of COVID-19. Cell, 181(5), 10361045.e9.

75. Carvelli, J., et al. (2020). Association of COVID-19 inflammation with activation of the C5a-C5aR1 axis. Nature, 588(7836), 146150.

76. Gao, T., et al. (2020) Highly pathogenic coronavirus $\mathrm{N}$ protein aggravates lung injury by MASP-2-mediated complement overactivation. medRxiv, 2020.03.29.20041962. 
77. Yu, J., et al. (2020). Direct activation of the alternative complement pathway by SARS-CoV-2 spike proteins is blocked by factor D inhibition. Blood, 136(18), 2080-2089.

78. Skendros, P., et al. (2020). Complement and tissue factor-enriched neutrophil extracellular traps are key drivers in COVID-19 immunothrombosis. The Journal of Clinical Investigation, 130(11), 6151-6157.

79. Mastellos, D. C., et al. (2020). Complement C3 vs C5 inhibition in severe COVID-19: Early clinical findings reveal differential biological efficacy. Clinical Immunology, 220, 108598.

80. Gao, Z. G., et al. (2013). The role of P2Y(14) and other P2Y receptors in degranulation of human LAD2 mast cells. Purinergic Signal, 9(1), 31-40.

81. Sala-Cunill, A., et al. (2015). Plasma contact system activation drives anaphylaxis in severe mast cell-mediated allergic reactions. The Journal of Allergy and Clinical Immunology, 135(4), 103143.e6.

82. Garvin, M. R., et al. (2020). A mechanistic model and therapeutic interventions for COVID-19 involving a RAS-mediated bradykinin storm. Elife, 9, e59177.

83. Nascimento, C. R., et al. (2017). Mast cell coupling to the kallikrein-kinin system fuels intracardiac parasitism and worsens heart pathology in experimental chagas disease. Frontiers in Immunology, 8, 840.

84. Scharfstein, J. (2018). Subverting bradykinin-evoked inflammation by co-opting the contact system: lessons from survival strategies of Trypanosoma cruzi. Current Opinion in Hematology, 25(5), 347357.
85. van de Veerdonk, F. L., et al. (2020). Kallikrein-kinin blockade in patients with COVID-19 to prevent acute respiratory distress syndrome. Elife, 9, e57555.

86. Motta Junior, J. D. S., et al. (2020). Mast cells in alveolar septa of COVID-19 patients: a pathogenic pathway that may link interstitial edema to immunothrombosis. Frontiers in Immunology, 11, 574862.

87. Wynn, T. A. (2015). Type 2 cytokines: mechanisms and therapeutic strategies. Nature Reviews. Immunology, 15(5), 271-282.

88. Yipp, B. G., et al. (2012). Infection-induced NETosis is a dynamic process involving neutrophil multitasking in vivo. Nature Medicine, 18(9), 1386-1393.

89. Gao, Z. G., Ding, Y., \& Jacobson, K. A. (2010). UDP-glucose acting at $\mathrm{P} 2 \mathrm{Y} 14$ receptors is a mediator of mast cell degranulation. Biochemical Pharmacology, 79(6), 873-879.

90. da Silva Ferreira, N. C., Alves, L. A., \& Soares-Bezerra, R. J. (2019). Potential therapeutic applications of P2 receptor antagonists: from bench to clinical trials. Current Drug Targets, 20(9), 919-937.

91. Mufti, F., et al. (2020). P2Y(14) receptor antagonists reverse chronic neuropathic pain in a mouse model. ACS Medicinal Chemistry Letters, 11(6), 1281-1286.

Publisher's Note Springer Nature remains neutral with regard to jurisdictional claims in published maps and institutional affiliations. 